\title{
HEADGROUP HYDRATION IN EGG-LECITHIN MULTIBILAYERS AFFECTS THE BEHAVIOUR OF DPH PROBES
}

\author{
H. VAN LANGEN, D. ENGELEN, G. VAN GINKEL and Y.K. LEVINE \\ Department of Molecular Biophysics, Physics Laboratory, University of Utrecht, \\ P.O.Box 80.000, 3508 TA Utrecht, The Netherlands
}

Received 18 February 1987 ; in final form 14 April 1987

\begin{abstract}
Angle-resolved fluorescence depolarization experiments were carried out on DPH molecules embedded in egg-licithin bilayers of different water contents. It was found that the order parameter $\left\langle P_{2}\right\rangle$ of the absorption moment is significantly higher than that of the emission moment and that the difference increases on hydrating the lecithin headgroups. The changes in the order parameter $\left\langle\boldsymbol{P}_{2}\right\rangle$ and $\left\langle\boldsymbol{P}_{4}\right\rangle$ of the DPH molecules induced by hydration are consistent with X-ray diffraction data. Furthermore the reorientational motions of the DPH molecules were found to become faster with increasing hydration.
\end{abstract}

\section{Introduction}

The probe molecule 1,6-diphenyl-1,3,5-hexatriene (DPH) is widely used in studies of the molecular order and dynamics of membrane systems with fluorescence depolarization tcchniques $[1,2]$. The molecule appears to behave as a cylindrically symmetric object and its absorption transition moment lies parallel to the long molecular symmetry axis. However, the excited-state properties of this molecule are not well understood. In particular experiments on DPH molecules embedded in macroscopically isotropic systems of membrane vesicles suspended in water suggest that the emission transition moment of the molecule also lies parallel to the symmetry axis $[3,4]$, while experiments utilizing macroscopically oriented membrane systems indicate that the two transition moments are not mutually parallel $[5,6]$.

The fluorescence anisotropy $r(t)$ is obtained by deconvolution procedures from the fluorescence emission observed from vesicle systems [7]. This quantity has been shown to be a sum over all the time correlation functions characterizing the reorientational motions of the probe molecules [8]. The limiting value of the anisotropy at time $t=0, r_{0}$, characterizes the mutual orientation of the two transition moments in the molecular frame. It is common practice to fix $r_{0}$ in the numerical analysis in order to facilitate the deconvolution procedures. The values of $r_{0}$ between 0.39 and 0.36 are obtained from depolarization experiments on viscous solutions of DPH in paraffin oil which indicate an upper limit of $15^{\circ}$ on the angle between the absorption and emission moments [9]. On the other hand, experiments on macroscopically oriented membrane systems afford the direct determination of the order parameters $S_{\mu}$ and $S_{\nu}$ of, respectively, the absorption and emission moments $[10,11]$. Here $S \equiv\left\langle P_{2}\right\rangle=$ $\frac{1}{2}\left\langle 3 \cos ^{2} \beta-1\right\rangle$ with $\beta$ the angle between the long molecular axis and the normal to the plane of the bilayer. We have previously shown that in a variety of lamellar model membranes $S_{\mu}$ is significantly higher than $S_{\nu}$, and angles of up to $35^{\circ}$ between the moments were obtained depending on the chemical composition of the system. This finding was corroborated by Johansson for DPH molecules embedded in oriented nematic lyotropic systems [12].

Here we show that for DPH molecules embedded in oriented multibilayers of egg-lecithin, the difference between $S_{\mu}$ and $S_{\nu}$ changes with increasing hydration of the headgroups of the lipids. Furthermore the changes in the molecular order and dynamics of the DPH molecules induced by the hydration are found to be consistent with the structural changes in the multibilayers obtained from X-ray diffraction experiments. 


\section{Experimental}

DPH was purchased from Fluka A.G. and was used as received. Egg-lecithin was obtained from Sigma. The preparation of the multibilayers was carried out as described previously [13]. The water content of the samples was varied by allowing the lipids to equilibrate with water vapour above a saturated salt solution for $24 \mathrm{~h}$. Saturated solutions of $\mathrm{K}_{2} \mathrm{SO}_{4}(98 \%$ relative humidity), $\mathrm{NaBr}(58 \%$ r.h.) and potassium acetate $(23 \%$ r.h. $)$ were used. The actual water content of the equilibrated samples was determined gravimetrically. The preparative steps were carried out as much as possible in the dark and under a nitrogen atmosphere.

The steady-state angle-resolved fluorescence depolarization experiments were carried out with a homebuilt instrument as described previously [5]. The sample plane was mounted vertically and the intensity ratio of light polarized perpendicular to and in the plane of incidence was determined. The depolarization ratios were measured for 56 different combinations of incidence and observation on excitation with light polarized in the plane of incidence.

The intrinsic fluorescence decay was obtained on exciting the sample under an angle of incidence in the sample of $\sin ^{-1}\left(3^{-1 / 2}\right)$ (i.e. $60^{\circ}$ in our case for $n=1.5$ ) with light polarized in the plane of incidence. The fluorescence emission along the normal to the sample surface is observed through a polarizer set with its optical axis at $45^{\circ}$ to the vertical. It can be easily shown from eq. (11) of ref. [10] that under these conditions the dynamical contributions to the fluorescence signal cancel out, so that only the intrinsic fluorescence decay is observed. This geometrical configuration for the determination of the fluorescence decay function is generally applicable.

The time-resolved measurements were carried out with the Daresbury synchrotron as a subnanosecond tunable light source operating with a repetition rate of $3 \mathrm{MHz}$. Single-photon counting techniques were used. The start pulse for the TAC (Ortec 457) was generated by the first photon detected by the PM tube (Philips XP2020Q) operating at $-2700 \mathrm{~V}$ and cooled to $-20^{\circ} \mathrm{C}$. The stop pulse was generated by the synchrotron ring pulse. Constant fraction discrimination (Ortec 463A) and delay units (EG\&G DB473) were used to define and delay the start and stop pulses. A multichannel analyzer (I/T Techno Inco. 5400) was used for $A / D$ conversion and for data collection of the TAC output. A minicomputer (PDP $11 / 04$ ) was used as an intermediate station between the MCA and the main frame computer. The excitation profile was measured by tuning the SPEX double-grating monochromator on the excitation side to the emission wavelength of $435 \mathrm{~nm}$. Light scattered from a very dilute solution of ludox, using the same scattering geometry as for the multibilayer sample, yielded the apparatus function. All the timeresolved measurements were completed within 10 $\min$.

All the experiments were carried out at room temperature, $21 \pm 2{ }^{\circ} \mathrm{C}$.

\section{Results and discussion}

Angle-resolved experiments were carried out on at least 3 samples of a given water content and the polarization ratios agreed within the experimental error of $2 \%$. The angle dependence of the polarization ratios affords the determination of five independent quantities $S_{\mu}, S_{\nu}, g_{0}, g_{1}$ and $g_{2}[10]$. Here $S_{\mu}$ and $S_{\nu}$ are respectively, the second-rank order parameters $\left(\equiv\left\langle P_{2}\right\rangle=\frac{1}{2}\left(\left\langle 3 \cos ^{2} \beta-1\right\rangle\right)\right.$ of the absorption and emission moments. The quantities $g_{k}, k=0,1,2$ are defined by

Table 1

The parameters characterizing the fluorescence from DPH molecules embedded in multibilayers of egg-lecithin with different water contents

\begin{tabular}{lclll}
\hline & $8 \%$ & & $10 \%^{\text {a) }}$ & $24 \%^{\text {a) }}$ \\
\hline$S_{\mu}$ & 0.61 & $( \pm 0.01)$ & 0.58 & 0.46 \\
$S_{\nu}$ & 0.58 & $( \pm 0.01)$ & 0.53 & 0.36 \\
$\beta_{\nu}(\mathrm{deg})$ & 12 & $( \pm 2)$ & 15 & 21 \\
$g_{0}$ & 0.42 & $( \pm 0.01)$ & 0.37 & 0.23 \\
$g_{1}$ & 0.062 & $( \pm 0.001)$ & 0.049 & 0.043 \\
$g_{2}$ & 0.018 & $( \pm 0.001)$ & 0.017 & 0.021 \\
$\alpha$ & 0.189 & $( \pm 0.005)$ & 0.0792 & 0.0592 \\
$\tau_{1}(\mathrm{~ns})$ & 3.6 & $( \pm 0.1)$ & 2.1 & 3.1 \\
$\tau_{2}(\mathrm{~ns})$ & 7.6 & $( \pm 0.1)$ & 7.4 & 7.9 \\
$\langle\tau\rangle$ (ns) & 6.8 & $( \pm 0.1)$ & 7.0 & 7.6 \\
\hline
\end{tabular}

a) Experimental errors are the same as given between parentheses for $8 \%$ water content. 
$g_{k}=\int_{0}^{\infty} G_{k}(t) F(t) \mathrm{d} t$,

$G_{k}(t)=\left\langle D_{k 0}^{2}\left(\Omega_{\mu}\right) D_{k 0}^{2}\left(\Omega_{\nu}\right)\right\rangle$,

$$
\begin{aligned}
& F(t)=\left(\alpha / \tau_{1}\right) \exp \left(-t / \tau_{1}\right) \\
& \quad+\left[(1-\alpha) / \tau_{2}\right] \exp \left(-t / \tau_{2}\right),
\end{aligned}
$$

where $D_{k l}^{2}$ are Wigner rotation matrix elements [14] and $\Omega_{\mu}$ and $\Omega_{\nu}$ denote respectively the orientation of the absorption moment at time $t=0$ and that of the

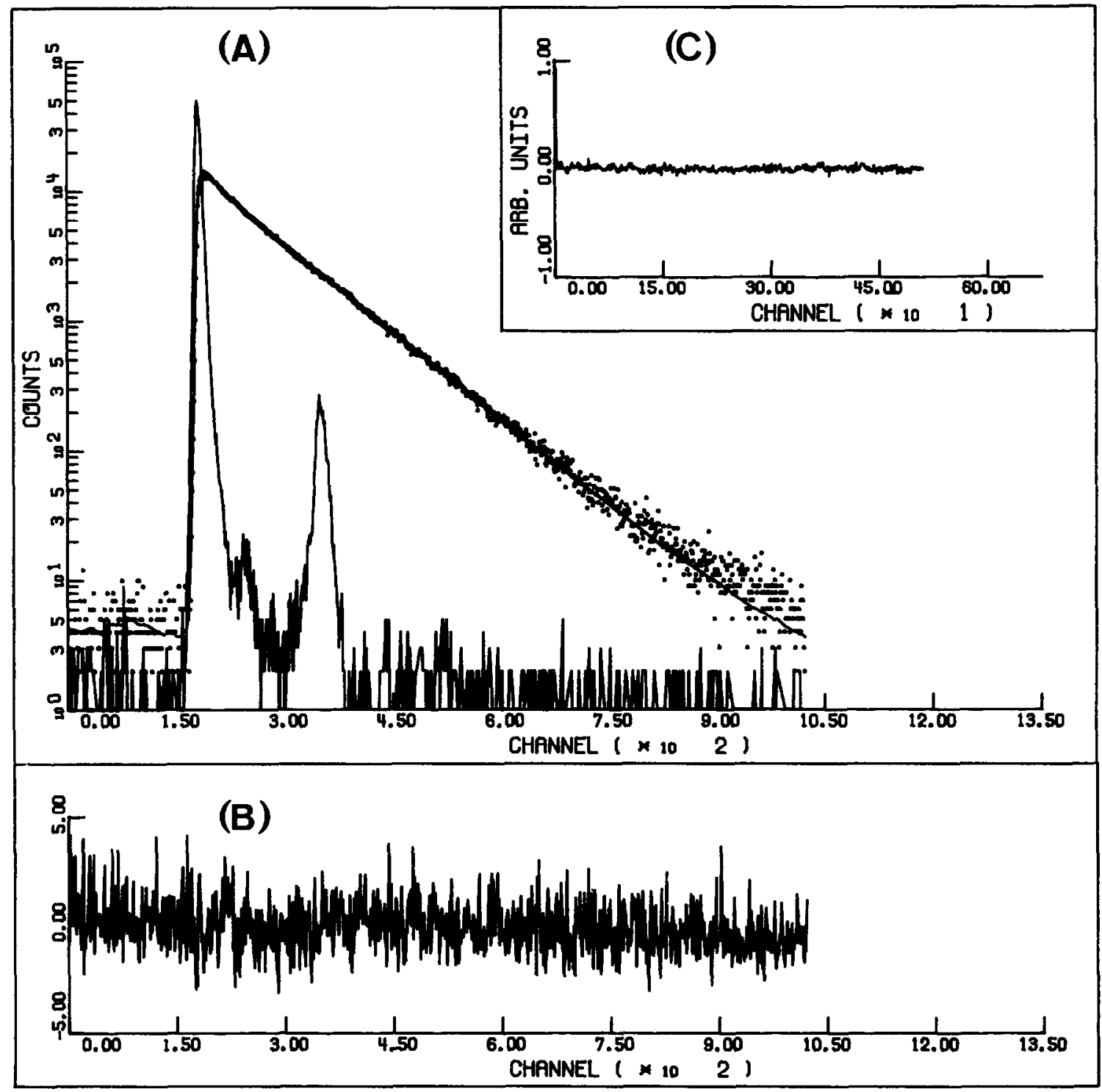

Fig. 1. The fluorescence decay for DPH in egg-lecithin bilayers with $10 \%$ water content. (A) shows the experimental points and the fitted line of the fluorescent decay. The narrow excitation pulse and the PMT after-pulse are also shown. One channel equals 78 ps. (B) and (C) show the residuals of the fit and the autocorrelation of these residuals respectively. 
emission moment at time $t$ in the bilayer-fixed reference frame. $F(t)$ is the normalized intrinsic fluorescence decay function.

The values of these parameters are shown in table 1. It is clear that for every system $S_{\mu}>S_{\nu}$, but that the difference decreases on reducing the hydration of the lipid headgroups.

These values of $S_{\mu}$ and $S_{\nu}$ are found to be independent of the wavelength of excitation in the 320-375 nm spectral region. On taking the DPH molecules to be cylindrically symmetric with the absorption transition moment $\mu$ lying along the long molecular axis we find [10]

$S_{\mu}=\left\langle P_{2}\right\rangle$.

Furthermore, if the emission transition moment $v$ is tilted by an angle $\beta_{\nu}$ with respect to the molecular axis, we have

$S_{\nu}=P_{2}\left(\cos \beta_{\nu}\right)\left\langle P_{2}\right\rangle$.

We have previously argued [13] that the latter assumption is consistent with the known photophysical behaviour of DPH. We thus find, table 1 , that $\beta_{\nu}$ increases from $12^{\circ}$ to $22^{\circ}$ on increasing the water content of the sample from $8 \%$ to $24 \%$.

The hydration dependence of the properties of DPH embedded in egg-lecithin bilayers is also reflected in the intrinsic fluorescence decay function. This function was obtained by fitting the experimental data using reiterative least-squares deconvolution procedures [7]. The best fit, as judged by the $\chi^{2}$ test, distribution of residuals and their correlation function, was obtained for a biexponential decay in agreement with measurements on vesicle systems [15]. This is shown in fig. 1. A small contribution from elastic scattering is present as can be seen from the residuals in the channels near the maximum of the excitation pulse. The decay parameters are shown in table 1 . It is evident that the biggest changes occur in the parameters of the short lifetime component, so that the average decay $\langle\tau\rangle=$ $\int_{0}^{\infty} t F(t) \mathrm{d} t$ decreases on hydrating the lipid bilayers.

Information about the reorientational dynamics of the molecules as well as the fourth-rank order parameter

$\left\langle P_{4}\right\rangle=\frac{1}{8}\left\langle 35 \cos ^{4} \beta-30 \cos ^{2} \beta+3\right\rangle$

can be obtained from the quantities $g_{k}$ on using a
Table 2

The best-fit parameters obtained from the rotational diffusion model

\begin{tabular}{lllcc}
\hline & $8 \%$ & & $10 \%{ }^{\text {al }}$ & $24 \%{ }^{\text {a) }}$ \\
\hline$\left\langle P_{2}\right\rangle$ & 0.61 & $( \pm 0.01)$ & 0.58 & 0.45 \\
$\left\langle P_{4}\right\rangle$ & 0.31 & $( \pm 0.02)$ & 0.29 & 0.20 \\
$D_{\perp}\left(\mathrm{ns}^{-1}\right)$ & $0.028( \pm 0.004)$ & 0.038 & 0.044 \\
$\beta_{\nu}(\mathrm{deg})$ & 12 & $( \pm 2)$ & 15 & 22 \\
\hline
\end{tabular}

a) Experimental errors are the same as given between parentheses for $8 \%$ water content.

motional model. We have analysed the experimental data in terms of the Brownian rotational diffusion model [2] and the fluorescence decay function of table 1 . Here the DPH molecules are assumed to undergo small-step stochastic diffusion subject to the orienting potential $U(\beta)$,

$U(\beta)=-k T\left[\lambda_{2} P_{2}(\cos \beta)+\lambda_{4} P_{4}(\cos \beta)\right]$,

where $P_{L}(\cos \beta)$ is the Legendre polynomial of rank $L$.

Details of the calculations can be found elsewhere [6]. In the calculations $\lambda_{2}, \lambda_{4}, P_{2}\left(\cos \beta_{\nu}\right)$ and $D_{\perp}$, the diffusion coefficients of the long molecular axis, were used as free model parameters and the results are summarized in table 2 . We were unable to obtain a consistent description of the data with the so-called Maier-Saupe potential on setting $\lambda_{4}=0$ in eq. (4). This problem arises because the MS potential underestimates $\left\langle P_{4}\right\rangle$. It is, furthermore, interesting to note that the values shown in table 2 changed by around $3 \%$ on taking the fluorescence decay function to be monoexponential with a lifetime equal either to $\langle\tau\rangle$ or to $\tau_{2}$, table 2 . This indicates that our experiment is not sensitive to the short lifetime component of the fluorescence decay in agreement with results obtained in other lipid systems [5].

It is interesting to note in this context that the values of the order parameters $\left\langle P_{2}\right\rangle$ and $\left\langle P_{4}\right\rangle$ obtained for egg-lecithin bilayers containing $8 \%$ water are identical to those found for DPH embedded in fully hydrated bilayers of dimyristoyl lecithin bilayers at $35^{\circ}$ [5]. Yet in the latter system the angle $\beta_{\nu}$ was found to be twice as large as in the former.

The orientational distribution functions of the DPH molecules derived from the orienting potential are shown in fig. 2. It is clearly seen that on hydrat- 

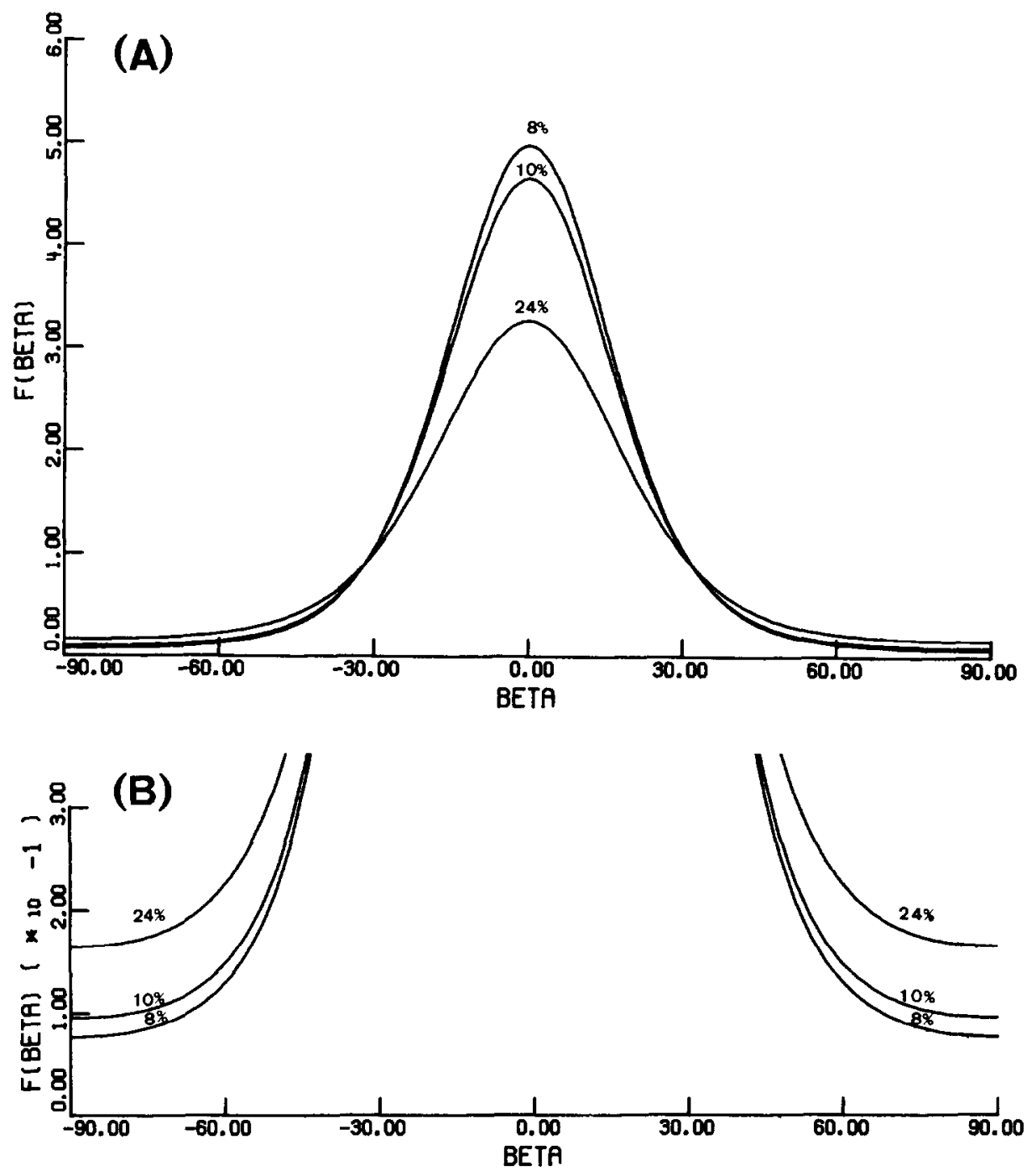

Fig. 2. The distribution function $f \beta$ ) is plotted (A) and enlarged (B) for the three different water contents.

ing the bilayers the distribution broadens and furthermore the probability of an orientation parallel to the bilayer surface $\left(\beta=90^{\circ}\right)$ increases significantly. This picture is consistent with $\mathrm{X}$-ray diffraction studies on egg-lecithin multibilayers, where it was found that the area per lipid molecule at the bilayer surface increases on hydrating the bilayers with a concomitant reduction in the degree of orientation of the long axes of hydrocarbon chains relative to the normal to the multibilayer plane [16].

Finally we note that the diffusion coefficient of the
DPH molecules increases significantly with increasing hydration. This behaviour correlates well with the observed changes in the angle $\beta_{\nu}$. However, such a correlation between $D_{\perp}$ and $\beta_{v}$ has not been found in previous results for fully hydrated lipid multibilayers [6].

\section{Acknowledgement}

This work was carried out with financial assis- 
tance from the Netherlands Foundation for Biophysics under auspices of the Netherlands Organization for Pure Research.

\section{References}

[1] K. Kinosita Jr., S. Kawato and A. Ikegami, Advan. Biophys. 17 (1984) 147.

[2] C. Zannoni, A. Arcioni and P. Cavatorta, Chem. Phys. Letters 32 (1983) 179.

[3] W.J. van Blitterswijk, R.P. van Hoeven and B.W. van der Meer, Biochim. Biophys. Acta 644 (1981) 323.

[4] C.D. Stubbs, K. Kinosita Jr., F. Munkonge, P.J. Quinn and A. Ikegami, Biochim. Biophys. Acta 775 (1984) 374.

[5] F. Mulders, H. van Langen, G. van Ginkel and Y.K. Levine, Biochim. Biophys. Acta 859 (1986) 209.

[6] G. van Ginkel, L.J. Korstanje, H. van Langen and Y.K. Levine, Faraday Discussions Chem. Soc. 81 (1987) 49.
[7] R.B. Cundall and R.E. Dale, Time-resolved fluorescence spectroscopy in biochemistry and biology (Plenum Press, New York, 1983).

[8] C. Zannoni, Mol. Phys. 42 (1981) 1303.

[9] R.E. Dale, L.A. Chen and L. Brand, J. Biol. Chem. 252 (1977) 7500 .

[10] B.W. van der Meer, R.P.H. Kooyman and Y.K. Levine, Chem. Phys. 66 (1982) 39.

[11] J.J. Fisz, Chem. Phys. 99 (1985) 177.

[12] L.B.-A. Johansson, Chem. Phys. Letters 118 (1985) 516.

[13] R.P.H. Kooyman, M.H. Vos and Y.K. Levine, Chem. Phys. 81 (1983) 461.

[14] M.E. Rose, Elementary theory of angular momentum (Wiley, New York, 1957).

[15] M. Ameloot, H. Hendrickx, W. Herreman, H. Pottel, F. van Cauwelaert and W. van der Meer, Biophys. J. 46 (1984) 525.

[16] Y.K. Levine and M.H.F. Wilkins, Nature 230 (1971) 69. 